livraisons

d'Histoire

de l'Architecture

\section{Livraisons de l'histoire de l'architecture}

$27 \mid 2014$

Varia II

\title{
Une robe nommée désir : contribution à la titrologie de mode dans l'entre-deux guerres
}

A Dress Named Desire: Contribution to the Titrology of Fashion during the Interwar Years

Kollektion Sehnsucht - ein Beitrag zur Benennung von Modeschöpfungen in der Zwischenkriegszeit

Johanna Zanon

\section{OpenEdition}

Journals

Édition électronique

URL : http://journals.openedition.org/lha/384

DOI : $10.4000 /$ lha. 384

ISSN : 1960-5994

Éditeur

Association Livraisons d'histoire de l'architecture - LHA

Édition imprimée

Date de publication : 10 juin 2014

Pagination : 129-152

ISSN : 1627-4970

Référence électronique

Johanna Zanon, « Une robe nommée désir : contribution à la titrologie de mode dans l'entre-deux guerres », Livraisons de l'histoire de l'architecture [En ligne], 27 | 2014, mis en ligne le 10 juin 2016, consulté le 19 avril 2019. URL : http://journals.openedition.org//ha/384 ; DOI : 10.4000//ha.384 
Par Johanna ZANON

\title{
UNE ROBE NOMMÉE DÉSIR : CONTRIBUTION À LA TITROLOGIE DE MODE DANS L'ENTRE-DEUX-GUERRES
}

\author{
«Yamilé, Zouave, Praline, Reine de Sabat, Platinum... Pourquoi \\ Platinum?"
}

Louis Roubaud, Au pays des mannequins, 1928.

La science des titres ou titrologie s'est développée à partir des années 1960 avec l'ouvrage fondamental de Michel Butor Les Mots de la peinture ${ }^{1}$ avant de connaittre un véritable essor dans les années 1980. Si le champ d'étude des titres littéraires et artistiques s'est étendu à la linguistique sous l'impulsion de Bernard Bosredon et à la sémiologie appliquée à l'histoire de l'art avec les travaux de Marianne Jakobi ${ }^{2}$, la question du titre de mode reste entièrement à défricher. L'objectif de cet article est de soulever ses enjeux par le prisme des appellations données par la maison de couture Jean Patou dans l'entre-deux-guerres.

Longtemps restée dans l'ombre de sa célèbre rivale Gabrielle Chanel, la maison fait pourtant partie des plus importantes de la période. Fondée en juin 1914 par Jean Patou, sa maison éponyme connaît un âge d'or dans les années 1920, où elle élabore son identité, expérimente des pratiques inédites et pose ses principes esthétiques. Dans le sillage de la crise de 1929, l'entreprise doit faire face à des difficultés financières croissantes. Le décès du couturier en 1936 marque la fin d'une époque de cette maison qui fermera ses portes bien plus tard, en 1987. Dans l'entre-deuxguerres, Jean Patou se démarque de ses concurrents par une politique de communication offensive, qui s'étend de la réclame traditionnelle insérée dans la presse spécialisée à la dynamique du scandale que suscitent ses défilés. La présentation des modèles à la clientèle s'accompagne d'une littérature commerciale, à décliner en cartons d'invitation, livrets d'accompagnement et livrets de défilé, où sont répertoriés les titres des modèles. Chaque collection de la maison Patou compte environ 300 modèles, à raison de deux grandes saisons annuelles, portant à 7200 le nombre de modèles - et donc de titres de mode - entre la réouverture au lendemain de la Première Guerre mondiale et 1936 . Ce corpus théorique se heurte toutefois aux aléas de la conservation : seuls 2000 titres de modèles ont été retrouvés grâce à six

1. Michel Butor, Les Mots dans la peinture, 1969, Genève, Skira, 181 p.

2. Bernard Bosredon, Les Titres de tableaux, une pragmatique de lidentification, 1997, Paris, PUF linguistique nouvelle, 274 p. ; Marianne Jakobi, Jean Dubuffet et la fabrique du titre, 2006, Paris, CNRS éd., 217 p. 
livrets de défilé ${ }^{3}$. Les dessins et photographies prises au titre de la protection de la propriété industrielle de la couture permettent d'éclairer cette source, de même que la presse spécialisée, friande de poésie vestimentaire. On postule que les titres de mode se situent à la croisée des genres entre titre artistique et argument commercial. Au-delà de l'évidente séduction féminine vantée par le titre, s'y concentrent à la fois la mode de la saison et l'identité de la marque.

\section{"Appeler un chat un chat" : titre / nom / sort}

Qualifier la parole attachée aux modèles de couture de titre de mode interroge le statut même de la couture. Le titre, du latin titulus, a une fonction originelle utilitaire : il désigne et identifie l'objet auquel il se rapporte. Jean-Paul Bouillon rappelle dans sa définition que "le titulus sert à introduire dans l'image qui, seule, resterait muette ou trop peu explicite la signification que le spectateur doit prêter à la représentation de tel sujet sous telle forme donnée ${ }^{4}$ ", annonçant la vocation moderne du titre, c'est-à-dire donner du sens à son objet. D'un point de vue typographique, l'italique sera donc préféré aux guillemets. L'analogie entre les titres de mode et les titres littéraires et artistiques replace au centre le débat entre art majeur et art mineur ou en d'autres termes la question de savoir si la couture peut être considérée comme un art. Le débat se cristallise dans l'entre-deux-guerres autour de la lutte contre la contrefaçon. Arguant de la théorie de l'unité de l'art, les couturiers se réclament des lois de 1793 relatives à la protection littéraire et artistique afin de protéger leurs modèles. Mais on pourrait aussi supposer qu'user de l'expression titre de mode réévalue la question à la façon des ready-made qui gagnent leur entrée au rayon de l'art notamment au moyen de leur titre : en 1917 un urinoir se change en Fontaine ${ }^{5}$ (ill. 1).

Un titre implique nécessairement un titreur. Le couturier choisit-il chaque titre de chaque modèle de sa collection ou est-ce un acte collectif ? Si l'on en croit la dernière chronique publicitaire qu'il écrit pour Votre Beauté et qui paraîtra à titre posthume en avril 1936, Jean Patou succomberait au plaisir de titrer comme l'appelle Françoise Armengaud dans ses entretiens : "J'ai réussi cette année une très belle robe [...] et j'en suis d'autant plus fier que j'avais appelé d'avance ce modèle : 1936, ce qui, à mes yeux, voulait indiquer qu'il était, par sa coupe et son tissu, exactement ce que l'on peut désirer actuellement ${ }^{6} »$. On note ici que le titre précède la robe. Jean Giono en expliquant la genèse de ses titres de roman affirme qu' « il faut un titre, parce que le titre est cette sorte de drapeau vers lequel on se

3. On a utilisé cinq livrets de défilé conservés à la Bibliothèque Forney (ca. hiver 1922 ; été 1929 ; hiver 1929-1930; été 1930 ; été 1931), cote CC2404, et un livret de défilé appartenant à une collection particulière (hiver 1927).

4. Jean-Paul Bouillon, "Titre des œuvres d'art ", Encyclopédie Universalis, 2002, p. 4557.

5. Marcel Duchamp, Fontaine, 1917. Voir Didier Semin, "Titre des ready-made", Encyclopédie Universalis en ligne.

6. «Ce que j'en pense... par Jean Patou ", Votre Beauté, avril 1936, p. 57. 


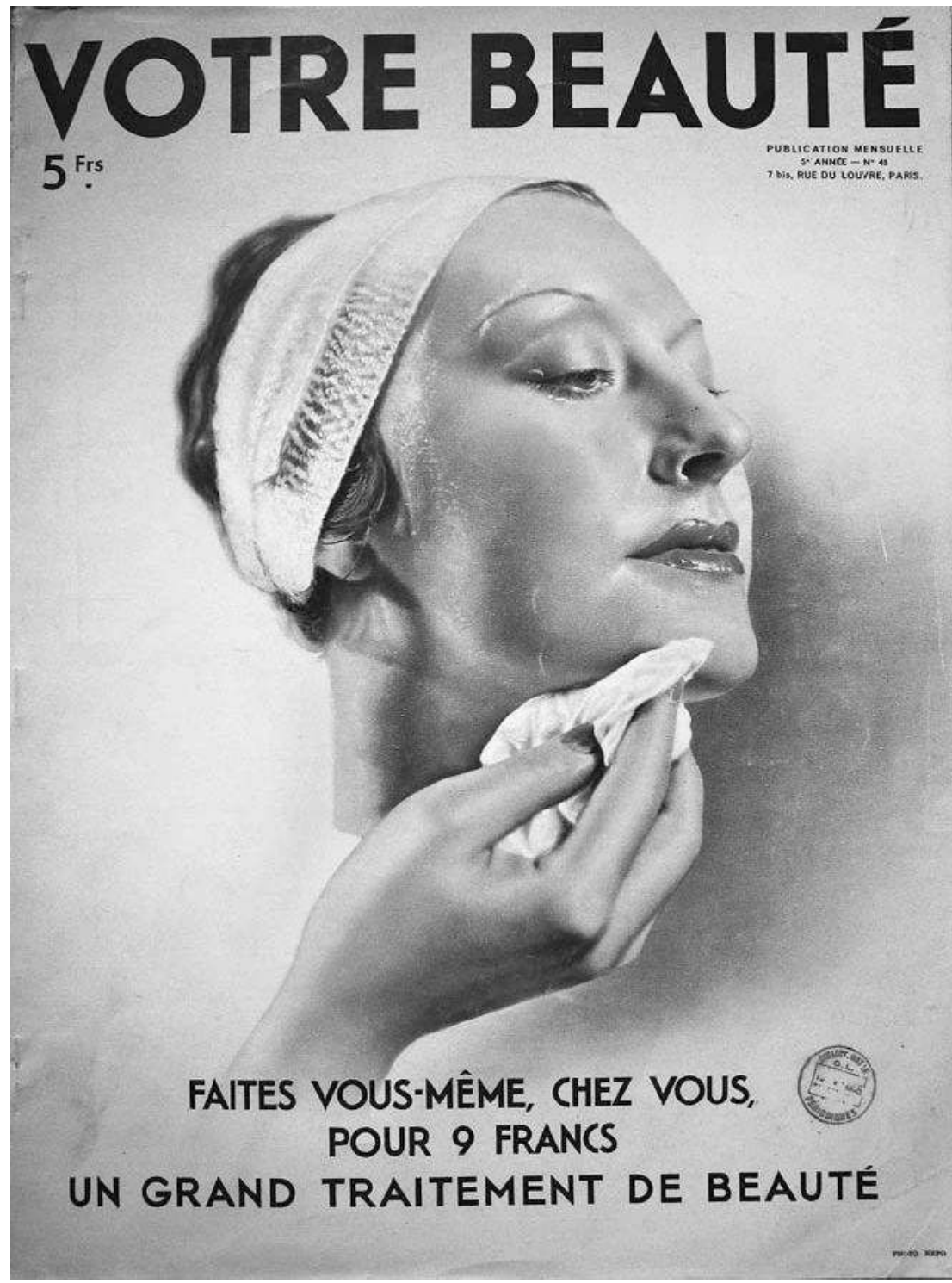

Ill. 1 : «Ce que j’en pense... par Jean Patou », Votre Beauté, avril 1936, BnF, Cl. Johanna Zanon.

dirige; le but qu'il faut atteindre, c'est expliquer le titre ${ }^{7}$ \%. Déterminer les trois cents titres que comporte une collection requiert une grande inspiration, aussi estil plus raisonnable de penser que le titre relève, le plus souvent, d'un acte collectif.

7. Cité par Gérard Genette, Seuils, 1969, Genève, Skira, p. 65. 
Le journaliste Louis Roubaud relate en 1928 sous forme de récit le choix du titre d'une robe de thé qui, de $A u$ temps de Joséphine, en référence au style Empire, devient $A u$ temps de Joséphine Baker ${ }^{8}$ pour marquer l'influence de l'art primitif en lien avec la Revue nègre.

L'auteur nomme cet acte rituel "baptême"; si l'on file la métaphore le(s) titreur(s) se mue(nt) en parrain. Le nom fait écho à la dimension religieuse, pour ne pas dire sacralisée, omniprésente dans la couture depuis Zola, et qui se retrouve encore chez la princesse Bibesco dans Noblesse de robe paru en 1928. La maison de couture représente l'église, le défilé la messe, le salon d'essayage le confessionnal ${ }^{9}$. C'est par le baptême qu'un assemblage de chiffons se transforme en modèle de couture : la mise en mot de la robe lui donne sa légitimité. Le modèle ne commence son existence publique qu'à partir du défilé. Lors de celui-ci, le nom est prononcé à haute voix, soit par le mannequin qui le porte, soit par une crieuse qui fait office de chef d'orchestre, soit par le couturier en personne comme chez Lucile $^{10}$. À l'appel du défilé, toutes les robes répondent présent. Leurs titres sont déclamés à la manière d'un poème, d'une litanie. Cette oralisation leur conferre une nouvelle dimension, qui n'est plus mystique mais magique. Le titre de mode est un sort jeté pour ensorceler la cliente : le modèle est invoqué lors de cette séance de spiritisme qu'est le défilé, elle prend corps dans toute sa matérialité.

Il est vrai que les titres ou noms de mode sont des "objets linguistiques étranges ${ }^{11}$ " selon les termes de Bernard Bosredon, qui explore l'intimité du lien qui unit un titre à sa toile. En de nombreux points, les titres s'apparentent à des noms propres bien qu'ils n'en aient pas la forme. Certains sont ainsi de véritables phrases: Viendra-t-il ? désigne une robe manteau à l'été 1922. Le titre, par sa qualité syntaxique de nom propre, individualise la robe au sein de la collection et de la catégorie de vêtements à laquelle il appartient : tailleurs du matin, tailleurs d'après-midi, robes tailleur, robes manteaux, manteaux d'auto, manteaux d'aprèsmidi, manteaux du soir, robes trois pièces, robes de thé, robes de dîner, robes du soir, pyjama-déshabillés à l'hiver 1922. En ce sens, le titre de mode rejoint le toponyme ou le patronyme, "par nature, individualisant" comme l'énonce Claude Lévi-Strauss ${ }^{12}$. Son existence s'opposerait à l'uniformité relative de la mode à la garçonne des années 1920, puis à la silhouette glamour des années 1930. Il consiste en une domestication et une appropriation des tendances de la mode. L'onomastique commerciale comme l'anthroponymie est sujette à la popularité fluctuante de certaines appellations; certaines ne durent qu'une collection tandis que d'autres reviennent saison après saison. Le titre de mode, en tant que nom propre,

8. Louis Roubaud, Au pays des mannequins : le roman de la robe, 1928, Paris, Les éditions de la France, 225 p., p. 48.

9. Cité par Rose Fortassier, Les Écrivains français et la mode de Balzac à nos jours, 1988, Paris, PUF, 233 p., p. 211.

10. Lady Duff Gordon, Discretions and Indiscretions, 1932, Londres, Jarrolds Ltd., 288 p., p. 187.

11. Bernard Bosredon, op. cit., p. 3.

12. Claude Lévi-Strauss, La Pensée sauvage, 1962, Paris, Plon, 395 p., p. 289. 
humanise la robe et contrebalance ainsi la déshumanisation du mannequin, cette poupée désincarnée. Les rôles se renversent, le mannequin perd son nom pour adopter celui de la robe: "Au bord du lac tourne la tête, s'arrête, repart, retourne et s'en va. Acacia la remplace ${ }^{13}$ ». La robe sort de l'anonymat. On peut postuler que l'apparition du titre de mode va de pair avec une société qui fait de plus en plus de place à l'individu.

\section{À la recherche du titre perdu: le titre comme annexe}

Le besoin d'intituler coïncide avec l'explosion du nombre de modèles dans chaque collection. À l'été 1930, Jean Patou n’en présente pas moins de 460. Nommer obéit dès lors à une nécessité pragmatique d'identification. Il faut mettre de l'ordre dans la profusion, fixer les limites de la multitude, définir la variation et le changement. C'est parce qu'il y a évolution qu'il y a nomination. Mais les titres de mode ont une vie fugace, plus encore que le modèle auquel ils sont liés. Très vite, ils tombent dans l'oubli. Près de 4000 se sont évaporés. Le titre n'est pas physiquement inscrit sur le modèle : il est énoncé, il est écrit sur le livret de défilé, il est relayé par la presse. Ni dessus, ni dedans, il est à côté. Le titre remplit le même rôle qu'un cadre : il entoure la robe. En suivant Gérard Genette, on peut dire que le titre de mode fait partie du métadiscours ou du " paratexte ${ }^{14}$ ». Dès lors qu'il a perdu sa fonction utilitaire et commerciale, le titre est relégué aux marges de la mode, déclassé, rejeté. Les noms errent sans corps, coquilles vides ; les modèles sans titre sont de mystérieux Médors ${ }^{15}$, comme tous les chiens perdus. "Des images et des mots sans bouteille. Jetés ensemble à la $\operatorname{mer}^{16}$ ", rêve l'artiste Pierre Alechinsky. Des listes de titres témoignent, seules, de l'existence de robes disparues. C'est le cas des livrets de défilé. Au contraire, la très grande majorité des robes photographiées au titre de la propriété industrielle sont retombées dans l'anonymat. Les vingt-et-une robes déposées le 27 août 1924 font figure d'exception, à l'instar du manteau du soir, répondant au nom de "Fatimah », et légendé de la sorte:

FATIMAH

256 - MANTEAU

Manteau du soir en velours de soie vert - broderie de perles, de strass, de cristal et d'argent sur le dos et le devant. Manches unies garnies de fourrure qui part en bande au dessous de bras et se termine en large parement - col et bande de fourrure ${ }^{17}$ (ill. 2).

13. Louis Roubaud, op. cit., p. 10.

14. Gérard Genette, op. cit., p. 7.

15. Pierre Alechinsky, Titres et pains perdus, Paris, Denoël, 1965, n.p.

16. Pierre Alechinsky, loc. cit.

17. Jean Patou, Fatimah, n 256 (8), dépôt n 7766 du 27 août 1924, Archives de Paris, fonds des dessins et modèles, cote D12U10 279. 


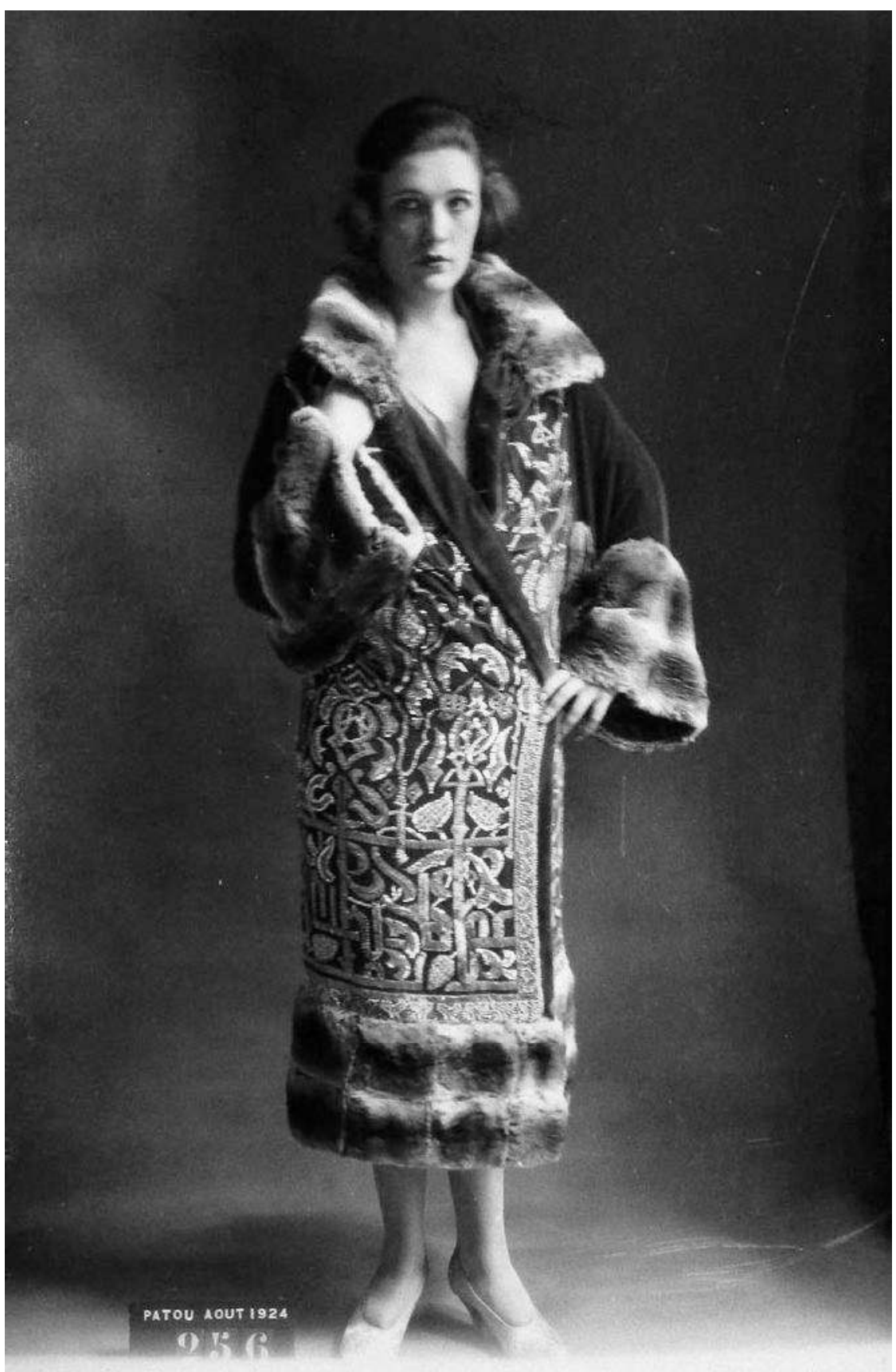

Ill. 2 : Jean Patou, Fatimah no 256 (8), dépôt no 7766 du 27 août 1924. Arch. de Paris, D12U10 279. Cl. Johanna Zanon. 
Dès lors, pourquoi ne pas les retitrer ? L'historien de la mode cherche à recréer le couple originel titre/modèle de couture. Il est rare cependant de trouver une description ou un visuel dans la presse de mode qui permette d'assortir à nouveau de façon valable un titre à son modèle - sauf à lâcher la bride à son imagination... Sur un mode poétique, c'est ce qu'a réalisé Olivier Saillard à l'occasion de l'exposition consacrée au chausseur Roger Vivier dans les espaces du Palais de Tokyo en 2013. Le livret d'aide à la visite précise: "Les titres et appellations assignés dans les vitrines sont des vues de l'esprit auxquelles les commissaires se sont attachés avec malice dans un souci scénographique ${ }^{18}$ ". La légèreté reprend ses droits après la disparition tragique des titres de mode. Les chaussures à talon sont affublées d'un autre titre, sorte de cognomen ou surnom. Arbitrairement, le commissaire a rebaptisé les chaussures en fonction des grands mouvements de l'histoire de l'art :

Dans une scénographie qui singe les musées du XIX ${ }^{\mathrm{e}}$ siècle, les chaussures épinglées comme des papillons ont pris la place des œuvres d'art consacrées. Les vitrines nobles au charme désuet dupliquent le vocabulaire docte du Prado et du Louvre. Des souliers, dont l'extraordinaire est le genre, ont préempté ces espaces et délogés les peintures et les sculptures d'avant. Talons "Étrave ", "Virgule», talon "Choc» se prennent pour des Vénus de bitume, des Joconde des parquets. Ils en ont le charme et l'arrogance ${ }^{19}$.

Ce surnom vient s'ajouter à la longue chaîne onomastique, débutée par le titre de mode, et qui s'enroule autour de chaque modèle. Il y a d'abord le nom de la maison, décliné en monogramme sur les tenues sportives de Jean Patou, et surtout ornant la griffe, cette signature du modèle. Sur celle-ci, on trouve aussi le nom des villes où sont installés des points de vente de la maison, avec une valeur d'appellation d'origine contrôlée. Viennent s'y greffer le nom du type de vêtement, le nom de la couleur, le nom des tissus qui le composent et dont s'amuse Colette dans $\mathrm{La}$ Fin de Chéri en 1926 : crépellaire, bigarelle, poplachan ${ }^{20} \ldots$, le nom du mannequin, le nom du photographe, le nom de la cliente, enfin le nom de l'institution de conservation. Les noms s'enfilent comme des perles pour former le collier, qui complète la tenue. Ils compensent le mutisme naturel de la robe. Michel Butor souligne que " notre regard est [...] tout préparé par un halo de commentaires ${ }^{21}$ ». Le titre est une surface qui affleure entre la mode et la littérature. Il est une zone de passage entre le couturier et le poète. Jean Patou est d'ailleurs un homme de l'écrit. Littéraire éclairé issu de la filière des humanités, il se passionne d'histoire, à tel point qu'il fait parâitre à ses frais un ensemble de douze lettres, jusque-là inédites, écrites de la main du marquis de La Fayette en 1780-1781 lors de la guerre d'Indépendance des États-Unis. En plus de sa collection d'art, il réunit une

18. Olivier Saillard (dir.), "Virgule, etc. Dans les pas de Roger Vivier », [Livret d'aide à la visite], Palais de Tokyo, du 2 octobre au 18 novembre 2013.

19. Loc. cit.

20. Cité par Rose Fortassier, op. cit., p. 176.

21. Michel Butor, op. cit., p. 6. 
importante bibliothèque allant de Charles Baudelaire à Rudyard Kipling. Au-delà, Roland Barthes s'interroge : "Les couturiers ne sont-ils pas des poètes qui écrivent d'année en année, de strophe en strophe, le chant de gloire du corps féminin ${ }^{22}$ ?»

Titreur des élites : ancrage socio-culturel du titre de mode

Les écritures titrologiques de Jean Patou se rangent en douze catégories formant le modèle onomastique de la maison de couture. Ce sont le désir et le sentiment amoureux ; la figure humaine et, particulièrement, la figure féminine; l'ancrage spatial; le mode de vie; la simplicité, la familiarité; la nature; la culture; la météorologie ; l'ancrage temporel ; la couture ; l'émotion. Le titre de mode a, avant tout, une visée commerciale, il est un argument de ralliement. Se crée ainsi une rhétorique de mode dans laquelle dominent les références au sentiment amoureux et à la féminité. Par sentiment amoureux, on entend le désir, la séduction, l'amour. Les titres de mode constituent une véritable carte du Tendre, où se situent à l'été 1931, Mon béguin, Pour lui plaire, Volage... De la même façon, la figure humaine est au centre des titres de mode de la maison Patou avec des noms de femmes anonymes ou célèbres, de personnages mythologiques, des odes à la féminité. On citera pêle-mêle pour l'été 1929: Antiope, Calypso, Circé, Colette, Corinne, Diane, Féminité, Femme fatale, Josette, Lisette, Médée, Miss Helyett, Nausicaa... Le titre incarne le désir de la cliente d'acheter la robe, le désir de l'homme pour la femme qui la porte. La figure masculine elle-même n'est pas absente, surgit ainsi à l'été 1929 le père du dandysme, Brummel. Peut-être faut-il y voir une volonté de travestissement, de masculinisation des femmes, en ces années folles où les Garçonnes règnent en maître. Désir, féminité, masculinité, tels sont les avatars de l'attractivité dans l'entre-deux-guerres. Le titre, pour faire vendre, véhicule des valeurs positives. Cela vaut aussi pour les titres qui retranscrivent une émotion: Happiness (1925), Bonne humeur (1927) Songerie (1929). Plus rarement, une pointe de nostalgie se fait jour, car Le bonheur passe, nous rappelle ce tailleur de 1921 (ill. 3).

Les titres de mode de Jean Patou affirment leur appartenance à l'époque au moyen d'un ancrage temporel, météorologique et géographique. Ils égrènent les saisons, Automne (1927), les heures du jour et de la nuit, Afternoon (1929) et les conditions météorologiques, Avant l'ondée (1929). Le titre donne un cadre au modèle, il le situe et le circonscrit. Ce désir de territoire s'exprime au travers d'une multitude de lieux, sorte de " géo-poétique ${ }^{23}$ " de la mode. La maison Patou donne à rêver à des contrées lointaines : Argentina (1923), Kaboul (1923), Kenitra (1924)... La dimension exotique prend d'autant plus de sens que la couture de l'entre-deuxguerres puise son inspiration à de nombreuses sources étrangères, à l'instar de l'ensemble des arts décoratifs. Le nom devient vecteur de voyage, il transporte en

22. Roland Barthes, Erté, 1973, Paris, Franco Maria Ricci, 189 p.

23. Martin de La Soudière, "Lieux dits : nommer, dé-nommer, re-nommer ", Ethnologie française, 1/2004, vol. 34, p. 70 . 


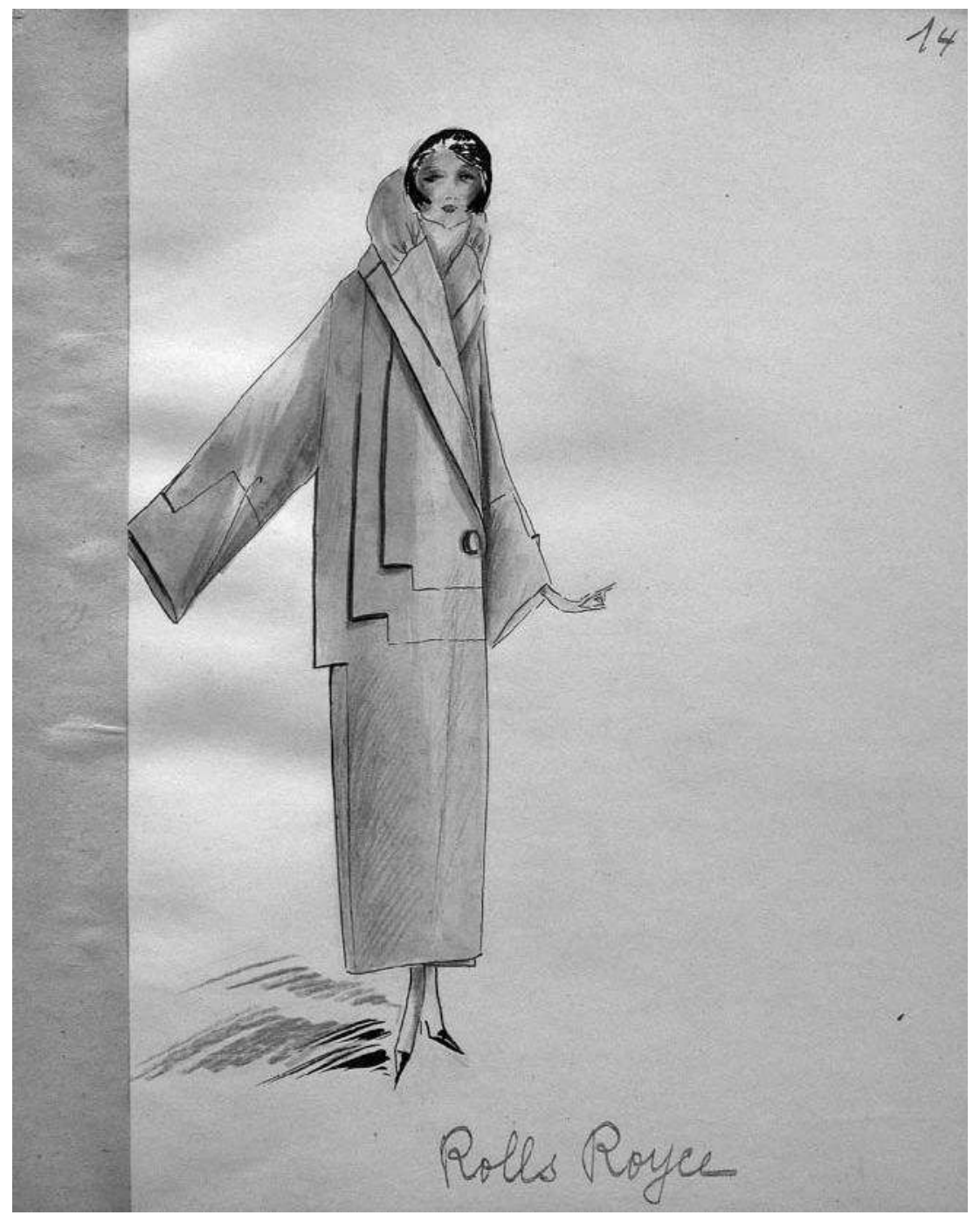

Ill. 3 : Jean Patou, Rolls-Royce, $\mathrm{n}^{\circ}$ 14, printemps 1922, 21,9 $\times 25,5 \mathrm{~cm}$, encre, crayon, aquarelle, collection particulière. Cl. Johanna Zanon.

imagination vers des paradis terrestres. Le lexique égrené au fil des titres s'en ressent, avec par exemple Départ et Dernier train à l'été 1929. L'ancrage géographique des titres témoigne aussi des lieux de sociabilité de la clientèle de Jean Patou, à commencer par les stations de ski, Chamonix (1927), et les stations balnéaires, La Baule (1929). Surtout, cet ancrage reflète les villes dans lesquelles le couturier a une succursale. Ses modèles s'intitulent alors Biarritz ou Cannes à l'hiver 1927. Certains titres, enfin, ont un lien métonymique avec les activités pratiquées par les élites ; 
c'est le cas des courses hippiques à Auteuil (1922) et à Longchamp (1929). Le milieu social de sa clientèle et le mode de vie correspondant est perceptible dans les titresmarques, ainsi choisis parce qu'ils inscrivent la maison de couture dans un paysage de consommation familier et haut de gamme. Rolls-oyce et Daimler titrent, en 1922, des manteaux d'auto.

La nature et la culture sont souvent représentées dans les titres de mode de la maison. Nombre de modèles de couture citent le monde floral, Campanule, végétal, Abricot, animal Corbeau, et minéral, Corail (1929). Les motifs floraux stylisés ont envahi l'Art Déco, ils ornent autant les robes que les grilles en fer forgé. La culture comprend les références à la littérature, comme Madame Bovary à l'été 1929 d'après le roman de Flaubert, mais aussi à l'art avec, notamment, La parisienne au bain, motif pictural traditionnel, ou Gioconda à l'été 1929. La référence au cinéma se fait jour dans un modèle comme Miarka (1922), d'après Miarka, la fille à l'ourse, un film muet de Louis Mercanton en 1920, adapté de l'œuvre de Jean Richepin. La culture populaire, avec le music-hall ou la chanson, interviennent dans le modèle onomastique de la maison Patou, quoique de façon plus sporadique. Ces références, souvent hermétiques pour le regard contemporain, sont le symbole d'une élégance sans chichis, d'une mode sobre et décontractée. Les surnoms, à l'image de Bobette en 1922, y font écho. Mamz'elle Fifi à l'été 1929, dont l'apostrophe possède un but évident d'oralité, l'exprime parfaitement. L'apostrophe commerciale, ainsi que le souligne Mat Pires, "dispose aussi d'une connotation propre, gage de bas prix, d'affaires à faire, et d'absence de prétention ${ }^{24}$ ». La simplicité est également une simplicité de mœurs ; la modestie et l'humilité sont des vertus toutes féminines en ces années 1920. Ce n'est pas le tailleur Sans prétention à l'été 1929 qui nous contredira. On peut, en outre, voir dans Mamz'elle Fifi un écho à la brièveté, à la rupture du rythme et donc à la syncope et au jazz. Et l'on pense inévitablement à Louis-Ferdinand Céline qui s'exclame dans Guignol's band : "Le Jazz a renversé la valse, l'Impressionisme a tué le "faux-jour", vous écrirez télégraphique ou vous écrirez plus du tout ! ${ }^{25} "$

S'il y a de la légèreté et de l'humour dans les titres de Jean Patou, nulle critique n'y transparaît. Marcel Duchamp raille cet argot de la couture, ou " poésie couturière " comme l'appelle Chanel avec dédain, en particulier celui de la parfumerie, en créant sous le pseudonyme Rrose Sélavy La Belle haleine, Eau de voilette en 1921. Elle opte d'ailleurs pour un numéro pour son plus célèbre parfum, le $N^{o} 5$. Car le refus de titrer en couture n'est jamais absolu : les numéros viennent se substituer aux mots pour identifier les modèles de couture. C'est le cas des premiers modèles du défilé de l'été 1931 chez Jean Patou. Parfois, il est vrai, les titres de mode ne sont pas très heureux. Trop fleuris, trop banals, trop évidents, trop faciles, les titres encombrent. Que penser chez Patou des Ciboulette, Jaune d'auf, Monsieur sans-gêne, Petite folle à l'été 1929 ? D'autres dénominations, au contraire, sont aptes

24. Mat Pires, "Leçons de Gram'hair : fonction de l'apostrophe en onomastique commerciale ", Langage et société, 1/2001, nº 91, p. 81.

25. Louis-Ferdinand Céline, Guignol's band, Paris, Gallimard, 1951, 377 p., p. 376-377.

Livraisons d'bistoire de l'architecture $n^{\circ} 27$ 
à persuader une grande partie des consommateurs. Les titres polysémiques en font partie. Le modèle Florentin (1922) désigne à la fois les habitants de Florence, un diamant exceptionnel aussi appelé Grand-duc de Toscane et porté disparu depuis 1922, des biscuits aux amandes et la rue de la maison de couture. Mat Pires précise que la polysémie est l'un des "piliers de la créativité publicitaire ${ }^{26}$ ", en ce que le client se délecte de l'énigme à résoudre.

La cliente doit deviner ce que représente le modèle qui défile devant ses yeux au moyen de son titre, qu'il soit "visuel " ou "idéel ${ }^{27}$ " selon la terminologie de Bernard Bosredon. Si le titre est visuel, cela est, somme toute, assez simple : son identité est "affichée ${ }^{28}$ ", comme le prouve Ruggero Druetta dans son étude de l'onomastique commerciale comme marqueur identitaire. La référence à la couture - couleur, matière fonction - participe de cette visualisation ${ }^{29}$. La jaquette Ragondin à l'hiver 1923 en est un exemple. Si le titre, en revanche, est indiciel ou idéel, la difficulté est plus importante: son identité est "révélée ». C'est ce que Françoise Armengaud nomme «l'effet-tilt ${ }^{30}$ ». Cela fonctionne chez Patou du fait de l'existence d'un même ancrage socio-culturel. Jean Patou n'est plus un "titreur d'élite ${ }^{31}$ ", selon le bon mot de Pierre Alechinksy, mais un titreur des élites. Ces élites aristocratiques, financières, sportives et cinématographiques, dont rend compte Emmanuelle Polle dans sa biographie du couturier ${ }^{32}$, peuvent être analysées au moyen des registres de clientèle, conservés dans les années 1920-1922. Le titre de mode est finalement un médiateur entre le groupe social de la clientèle et les vêtements qu'elle porte, le titre "confere à l'un comme à l'autre un surcroît d'identité $^{33}$ ». Il replace le modèle de couture dans son contexte de production, il est le témoin d'une époque : les années folles et les années 1930 paradent au fil des livrets de défilés. Le titre montre comment la maison Jean Patou s'est appropriée la mode saison après saison. En cela, le titre est un marqueur identitaire.

\section{Sentir l'invisible : spécificité de l'onomastique olfactive}

Les titres de parfum sont exempts de la profusion qui caractérise les titres de mode. Deux obstacles se dressent néanmoins sur leur route. Frappés de cécité, ils sont privés d'appui visuel. La stratégie de communication est contrainte à créer un univers de toute pièce. Le titre du parfum acquiert une valeur stratégique : il est le

26. Mat Pires, op. cit., p. 72.

27. Bernard Bosredon, op. cit., p. 10.

28. Ruggero Druetta, "Les noms de marque et de produit comme marqueurs identitaires ", Ela. Études de linguistique appliquée, 2008/2, n ${ }^{\circ} 150$, p. 157-175.

29. «Visualisation. Ensemble de traces linguistiques exprimant la visibilité du référent. La visualisation caractérise l'intitulation moderne de la peinture ", Bernard Bosredon, op. cit., p. 10.

30. Françoise Armengaud, Titres. Entretiens, 1988, Paris, Méridiens Klincksieck, 348 p., p. 14.

31. Pierre Alechinsky, Le Test du titre, Paris, Éric Losfeld, 1967, p. 2.

32. Emmanuelle Polle, Jean Patou, une vie sur mesure, 2013, Paris, Flammarion, 279 p.

33. Martin de La Soudière, op. cit., p. 72. 
point d'ancrage à partir duquel l'univers de marque va se créer. C'est un phare dans la nuit. Il permet de donner corps à l'immatériel, de mettre un nom sur une odeur volatile et, par nature, indicible. Ainsi en est-il de Normandie. Jean Patou profite du lancement du transatlantique pour intituler son nouveau parfum, distribué en avant-première aux voyageurs de la traversée inaugurale. Le flacon lui-même affiche sa filiation onomastique dans la forme de son flacon : il est enserré au centre d'un paquebot d'acier aux lignes stylisées. Le Normandie, qui appelle la mer et le voyage, justifie son titre d'après les chroniqueurs de l'époque :

Son homonyme est un parfum chaud, têtu, obstiné, qui fait songer à tous les symboles de l'élégance. Sa fraîcheur d'algues mouillées, d'écorces et de mousses méditerranéennes que lui donne une base chyprée, apporte l'accueil parfumé des escales de croisière. Ses touches de bois d'Orient, enfin, complètent la suggestion en vous transportant, si vous fermez les yeux, dans ces coffres de cèdre, d'acajou et de macassar verni, que sont souvent les plus belles cabines des transatlantiques ${ }^{34}$ (ill. 4).

Il colle ainsi à l'imaginaire contemporain suscité par ce fait d'actualité, sans réussir à le transcender pour en faire une création pérenne une fois l'attrait pour l'événement effacé. Seul le célèbre Joy y est parvenu. Les autres parfums misent principalement sur deux tendances : le jeu amoureux et l'ivresse bachique. Les trois premiers parfums lancés coup sur coup en 1925 - Que sais-je? Amour amour, Adieu sagesse - sont chacun destinés à un type de chevelure - brune, blonde, rousse - et correspondent aussi aux étapes d'un flirt - l'hésitation amoureuse, le moment où les battements de cœur s'accélèrent, quand la raison cède au cœur. La référence aux boissons alcoolisées transparait notamment dans deux coffrets de parfumerie, appelés Bar à parfum et Cocktail (1930), inspirés des bars américains comme celui du Ritz ouvert en 1921. Ils permettent à la cliente de mélanger les senteurs pour se composer une odeur unique. Cocktail comprend quatre parfums majeurs - Dry, Sweet, My Own, Bitter Sweet - et sept senteurs mineures, les Angostura. La terminologie de ces parfums reprend la taxinomie des spiritueux : le parfum, assimilé à l'alcool, est perçu dans sa dimension liquide et non gazeuse, évoluant de l'odorat vers le goût. L'imaginaire culturel bachique contribue à associer parfum et plaisir charnel. Un véritable bar a même été aménagé au premier étage de la maison témoin de la libération des mœurs après la Première Guerre mondiale :

Nulle part ensuite je n'ai vu autant de femmes aussi belles! Vous comprenez, c'étaient des femmes, venues du monde entier, qui ne vivaient que pour s'habiller. Le bar était ouvert aux hommes. Que de jolies scènes de séduc$\operatorname{tion}^{35}$...

34. Michel Arbaud, "Eaux de Cologne, Parfums d'été ", Votre Beauté, juin 1935, p. 46

35. Madeleine Chapsal, La Chair de la robe, 1989, Paris, Fayard, 377 p., p. 239.

Livraisons y'bistoire de l'architecture $n^{\circ} 27$ 


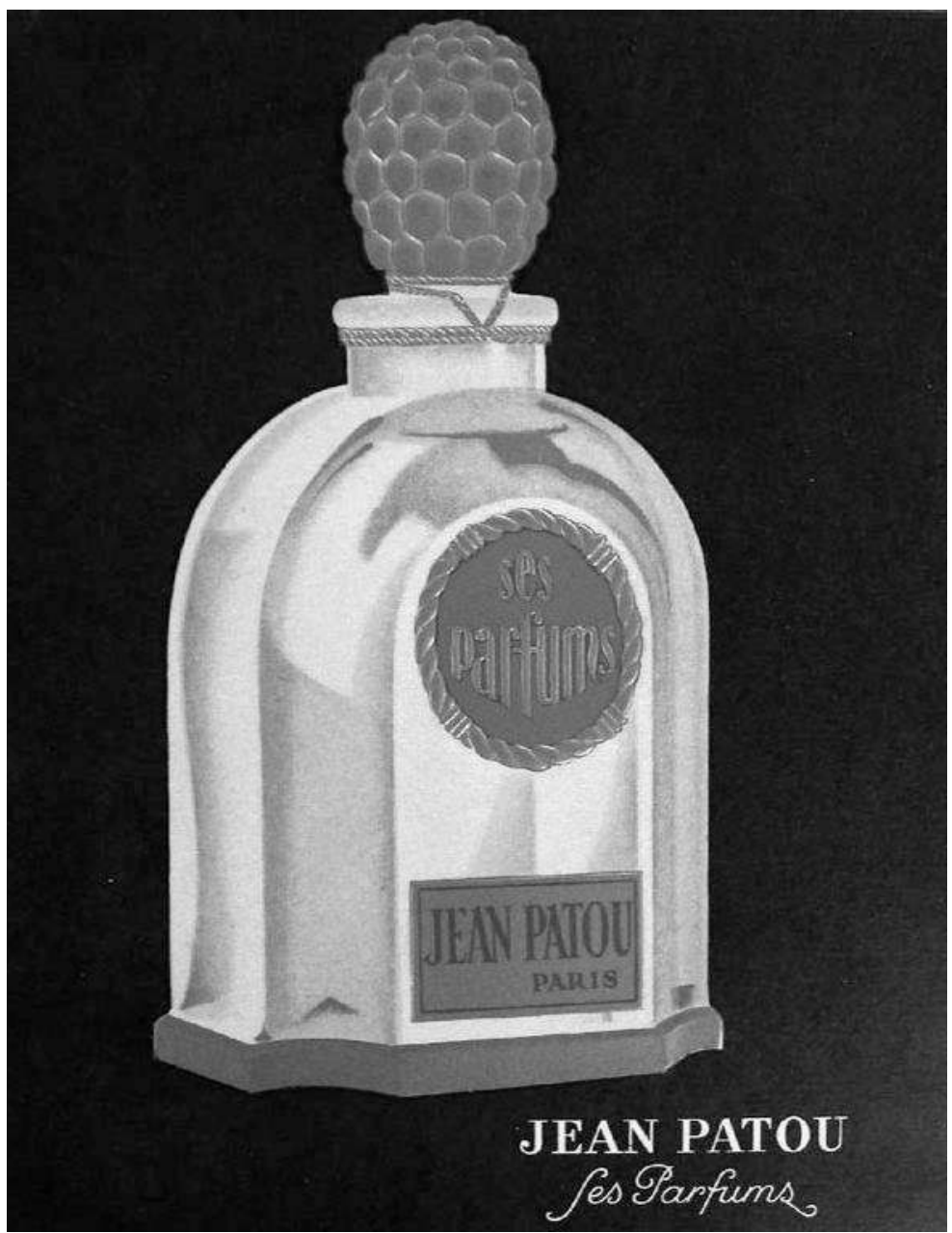

Ill. 4 : Jean Patou, Ses Parfums, 1925, Bibliothèque Forney, CC2404. Cl. Johanna Zanon.

La seconde pierre d'achoppement se matérialise dans la faiblesse de protection dont bénéficient les parfums. Il est impossible, en effet, de protéger la formule aromatique. Celle-ci est exclue des inventions brevetables, au même titre que les combinaisons pharmaceutiques et les remèdes. Il ne reste que le titre et le flacon pour se protéger. Si le flacon, dessiné par les architectes-ensembliers Louis Süe et André Mare et réalisé par Baccarat, est déposé sous forme de dessins et modèle au greffe du conseil de prud'homme, le titre se place sous la protection des marques de commerce et de fabrique. Jean Patou dépose méticuleusement la plupart de ses titres de parfums et de cosmétiques, même si certains y échappent comme le Lait Nordique en 1931 ou Invitation en 1932. A contrario, le couturier ne dépose aucune 
marque pour des titres de mode. La seule exception, anecdotique, concerne les robes intitulées comme les parfums de la maison, à l'exemple du manteau Amour amour à l'hiver 1927. Après le procès de Jeanne Paquin contre Beer à propos des modèles et marques Bellona, Edile et Belize, la jurisprudence a tranché qu'il n'était nul besoin que le modèle incriminé porte le même nom que le modèle original pour être attaqué en contrefaçon ${ }^{36}$ (ill. 5).

Le souci particulier accordé à la dénomination des parfums tient enfin au fait que les maisons de couture se diversifient dans cette activité pour assurer la survivance de leur nom. La parfumerie acquiert un fort capital mémoriel et, au premier rang, Joy (1930). La réussite mondiale et durable de cette fragrance tient à la réunion d'un produit, d'un titre, d'un slogan et d'une stratégie de diffusion. Joy est un produit d'exception qui se fonde sur un puissant accord rose-jasmin. Joy est un titre court, anglais, sans ambiguïté, qui s'inscrit dans l'esthétique de la litote. Facile à prononcer, facile à retenir, une syllabe suffit pour invoquer l'allégresse et conjurer le mauvais sort d'un fameux jeudi noir d'octobre 1929, qui voit s'effondrer la bourse new-yorkaise. "Joy est un enfant de la crise ${ }^{37}$ ", selon le parfumeur Jean Kerléo : il la tourne en dérision. Joy est vendu comme étant «le parfum le plus cher au monde", tournant son principal défaut en son meilleur argument de vente. L'image "excentrique personnalise $J y^{38}$ ", selon la spécialiste Mariette Julien, en rupture avec la psychologie traditionnelle du parfum. Il s'adresse aux femmes les plus aisées, mais sans distinction de couleur de chevelure cette fois. Il mise sur l'universalité de sa formule. Joy est avant tout destiné au marché américain : ce parfum est le Graal d'une quête olfactive menée par Jean Patou et la publiciste Elsa Maxwell pour s'implanter aux États-Unis. La diffusion de Joy correspond ainsi à l'institutionnalisation de la parfumerie avec la création de la filiale Jean Patou Inc. en 1930, avant même son homologue française en 1932, Jean Patou Parfumeur.

\section{Naming the dress : traduction du titre et version originale}

La nationalité de la clientèle et la prétention à la l'universalité des produits de la maison Jean Patou posent la question de la traduction des titres de parfums et de mode. Certaines marques de parfumerie, pour voyager outre-Atlantique, sont traduites en anglais de façon littérale. C'est le cas de Coquetel (1929) / Cocktail (1928), La joie de Jean Patou / Joy (1930), Happy Lovers / Heureux amants (1930), Cocktail de poudres / Powder cocktail (1931). La forte charge identitaire véhiculée

36. André Allart, Paul Carteron, La Mode devant les tribunaux, 1914, Paris, L. Tenin, Recueil Siret, 100 p., p. 33-36

37. Interview de Jean Kerléo dans le documentaire d'Emmanuelle Polle, Jean Patou (1887-1936) : une vie sur mesure, France Culture, 2009.

38. Mariette Julien, L'Image publicitaire des parfums, communication olfactive, 1997, Paris, L'Harmattan, 276 p., p. 47. 


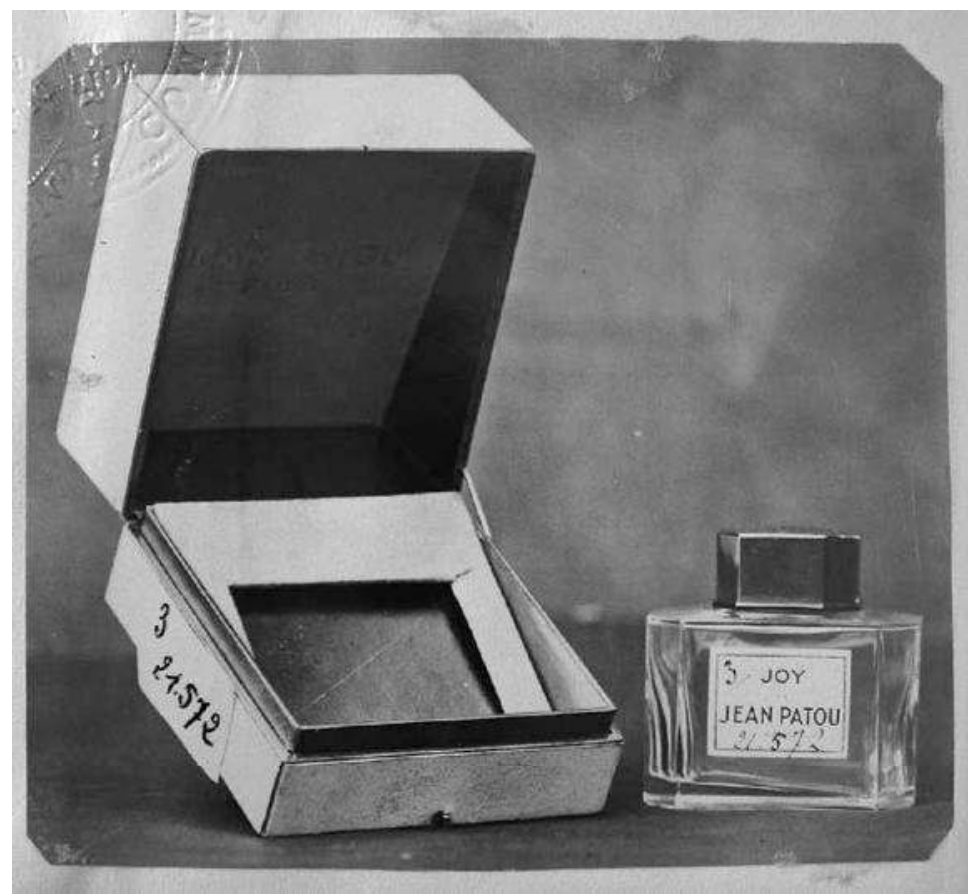

Ill. 5 : Jean Patou, Joy, octobre 1931, INPI, BOPI Dessins et modèles, registre 171, nº 21572, p. 76. Cl. Johanna Zanon.

par ces marques doit être aisément compréhensible par la clientèle cible. De la même façon que, comme le rappelle Christian Moncelet dans son Essai sur le titre, un film lorsqu'il change de pays change aussi de titre ${ }^{39}$, la maison de couture peut faire le choix de modifier le titre pour en préserver le sens. May Be (1925) n'est autre que la traduction libre de Que sais-je? qui explicite l'hésitation amoureuse pour qui ne maîtrise pas la langue de Molière. De même, Patou's Own fait écho à Le Sien (1928). Elsa Maxwell, qui s'occupait de la publicité chez Jean Patou, narre la genèse du processus de titulation de Joy:

Joie ? répéta notre parfumeur [Henri Alméras], traduisant en français.

Non, Joy. Le monde entier comprendra. Partout où l'on vend du parfum, Joy sera le synonyme de perfection, comme Rolls-Royce pour les automobiles ${ }^{40}$.

39. Christian Moncelet, Essai sur le titre en littérature et dans les arts, 1972, [édition à compte d'auteur], p. 197-198.

40. Elsa Maxwell, J'ai reçu le monde entier, Paris, Amiot-Dumont, 1955, p. 126. 


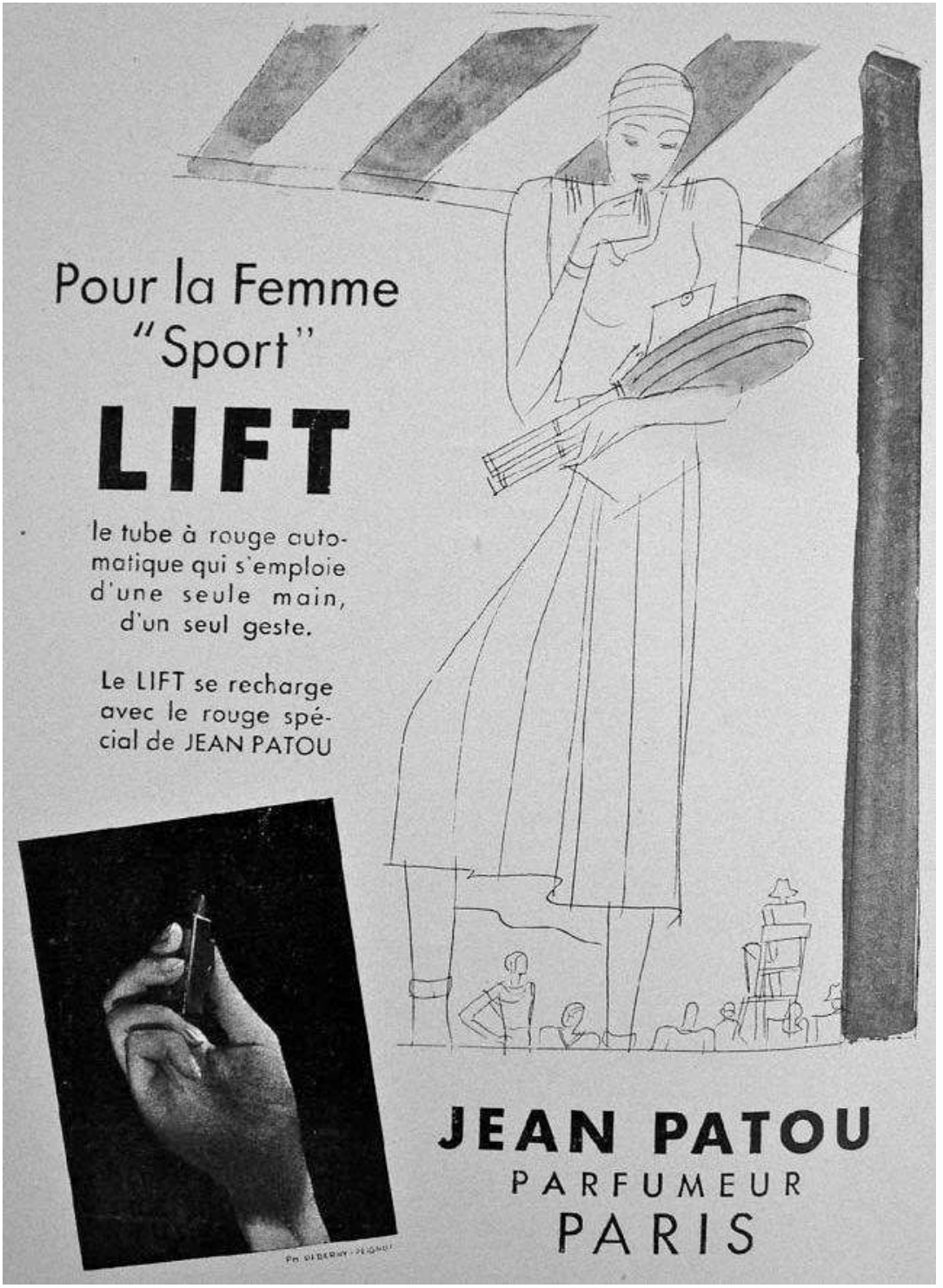

Ill. 6 : Jean Patou, publicité pour Lift, dans le Livret de défilé, été 1931, Bibliothèque Forney, CC2404. Cl. Johanna Zanon.

Si la traduction La joie de Jean Patou est tout de même déposée par prudence commerciale en août 1930, un mois seulement après la version anglaise, il n'en reste pas moins que tout le monde a compris la version originale, que les clientes soient anglophones ou francophones (ill. 6). 
La commercialisation de parfums ou modèles de couture français aux ÉtatsUnis et au Royaume-Uni ou à des clientes américaines et britanniques en France joue sur les stéréotypes associés à chaque culture comme le montre Ruggero Druetta dans son analyse des appellations commerciales italiennes, grecques et basques en France : «le succès et/ou l'insuccès tiennent à la langue, formidable instrument de communication et de ralliement identitaire, véritable supplément d'âme de cet être de paroles qu'est la créature humaine ${ }^{41}$. " L'emprunt à la langue anglaise reflète la modernité que projette en eux Jean Patou. Le rouge à lèvre Lift (1931) en est le meilleur exemple. Son titre veut dire ascenseur en anglais, en écho à son mécanisme, breveté en 1929 et qui fait l'objet de la première licence signée par la maison Patou, avec le joaillier Cartier. Toute la communication de ce rouge à lèvres est axée sur le mécanisme, à tel point que ses publicités singent un mode d'emploi. Chaque collection comporte également son lot de titres en anglais. À l'été 1929, on en recense 30 sur 387, dont Afternoon, Beauty, Black \& White, Blue Girl, Darling, Merry Girl, Patou's Eccentric Capucine, Play, Ready, Smash, Sunshine, Swimming...

L'usage de la langue anglaise revêt une signification particulière pour Jean Patou. Lui-même la parle mal ainsi que le spécifie son dossier militaire, néanmoins il entretient des liens forts avec le Nouveau Monde. En novembre 1924, il recrute six mannequins américains pour vanter les mérites de la Diane américaine par opposition à la Vénus française. L'audace de son geste ne manque pas de susciter une vive polémique. Caroline Evans, qui leur consacre un article, a aussi étudié à partir d'une robe de 1925, brodée d'un motif de Tour Eiffel, les regards croisés entre les États-Unis et la France révélés par la publicité chez Jean Patou, au travers des problématiques liées de mode et modernisme ${ }^{42}$. En France, Jean Patou est considéré comme moderne parce qu'utilisant les pratiques américaines, tandis qu’aux États-Unis, la Tour Eiffel résume la quintessence de son élégance parisienne. Les États-Unis exercent une réelle fascination sur Jean Patou l'amenant à tisser des liens privilégiés avec certains acheteurs professionnels américains, comme le grand magasin Bergdorf Goodman sur la $\mathrm{V}^{\mathrm{e}}$ avenue. Le fils du fondateur, Andrew Goodman, se forme même chez Patou en 1928. Pendant son séjour dans la maison, il infiltre et aide à démanteler un réseau de contrefacteurs ${ }^{43}$.

L'anglais n'est pas la seule langue étrangère présente dans les titres de mode. Changer de langue véhicule une notion de l'ailleurs et fait voyager vers d'autres paradis terrestres. Surtout, le titre en langue étrangère donne une nouvelle frấcheur : inhabituel à l'oreille, il retient l'attention de la cliente. «Donner un titre dans une langue étrangère, c'est encore une manière de rompre avec les connotations sédimentées de la langue [maternelle] ${ }^{44}$ ", affirme l'artiste Noël Dolla. Changer de langue permet aussi d'exprimer des notions et des nuances inconnues en

41. Ruggero Druetta, op. cit., p. 174.

42. Caroline Evans, "Jean Patou's American Mannequins : Early Fashion Shows and Modernism", Modernism/Modernity, The John Hopkins University Press, 2008, 15/2, p. 243-263 ; Caroline Evans, "Paris-New York 1925 : Jean Patou's “Advertising” ", dans Andrea Kollnitz, Louise Wallenberg (eds), Modernism and Fashion, Stockholm, Carlssons Bokförlag, en cours de publication.

43. Didier Grumbach, Histoires de la mode, 2008, Paris, Le Regard, 450 p., p. 96-97.

44. Cité par Françoise Armengaud, op. cit., p. 276. 
français. Pour ses titres, le plasticien Arman alterne entre l'anglais, le français et l'espagnol pour traduire au mieux sa pensée ${ }^{45}$. Chez Jean Patou, l'italien tient le second rôle dans la pièce de couture qui se joue à chaque saison. Perçue comme la langue de la séduction, ce que prouve le sensuel Caro Mio à l'hiver 1927, l'italien incarne le désir de la cliente. L'espagnol, le russe, l'allemand et certaines langues d'Europe de l'Est font aussi de brèves apparitions au fil des livrets de défilés de la maison.

\section{Inventaire à la Prévert: la longue liste des titres de mode (ill. 7)}

Les livrets de défilés recensent les modèles, en une sorte d'inventaire à la Prévert. Ainsi se suivent les robes de thé à l'hiver 1922: 60. Bonheur de Mag, 61. Claridge, 62. Confucius, 63. Delphine, 64. Désir de plaire, 65. Douce surprise, 66. Doux présage, 67. Échevelée, 68. Fausse Ingénue, 69. Florise... jusqu'au numéro 84. Vierge folle. Entre eux, nul lien direct n'apparaît : c'est une énumération hétéroclite, sans queue ni tête. Cette folle liste ne se cantonne pas aux livrets de défilé, elle envahit les comptes rendus de défilés dans la presse. L'Officiel de la couture cite quarante titres de mode pour le défilé de Jean Patou en août $1922^{46}$. Les titres accumulés les uns au-dessous des autres produisent dans les livrets de défilé un " effet-catalogue" selon la typologie établie par Françoise Armengaud. Bientôt, à partir de l'hiver 1927 à tout le moins, la maison met de l'ordre; l'énumération devient alphabétique, manière de dictionnaire sans définition. L'effet catalogue conserve son étrangeté déroutante. Idées roses, I love you, Il pleut bergère, Il viendra, Imbroglio, Imprévu, Inconnue, Intimité, Intrigante, Iris noir, Isabelle: la lettre "i " continue de sauter du coq à l'âne à l'été 1930. Ces amas de titres oscillent sans cesse entre les champs de l'utile et du poétique en une douce mélodie commerciale. Bien que finies, ces listes donnent à voir l'excès et la démesure des collections de la haute couture dans l'entre-deux-guerres. Le vertige de la liste, d'après le titre de l'essai d'Umberto Eco, y est palpable et il se poursuit sur le podium :

Ici, la succession de créatures ensorceleuses entend juste suggérer l'abondance, rassasier le besoin de kolossal, montrer non point une seule image fascinante mais beaucoup, plein, à foison, offrir à l'usager une réserve inépuisable d'appels voluptueux [...]. La technique de l'énumération n'entend pas ici mettre en doute l'ordre du monde, au contraire, elle veut réaffirmer que l'univers de l'abondance et de la consommation, à la disposition de tous, représente le seul et unique modèle d'une société ordonnée. L'énumération de diverses beautés a affaire avec les caractéristiques de la société engendrée par les mass media. Marx rappelait au début du Capital que « la richesse des sociétés dans lesquelles règne le mode de production capitaliste s'annonce comme une "immense accumulation de marchandises" 47 ".

45. Ibid., p. 70.

46. L'Officiel de la couture, de la mode et de la confection, $\mathrm{n}^{\circ} 13$, août 1922 , p. 9 et 11.

47. Umberto Eco, Vertige de la liste, 2009, Paris, Flammarion, 408 p., p. 253. 


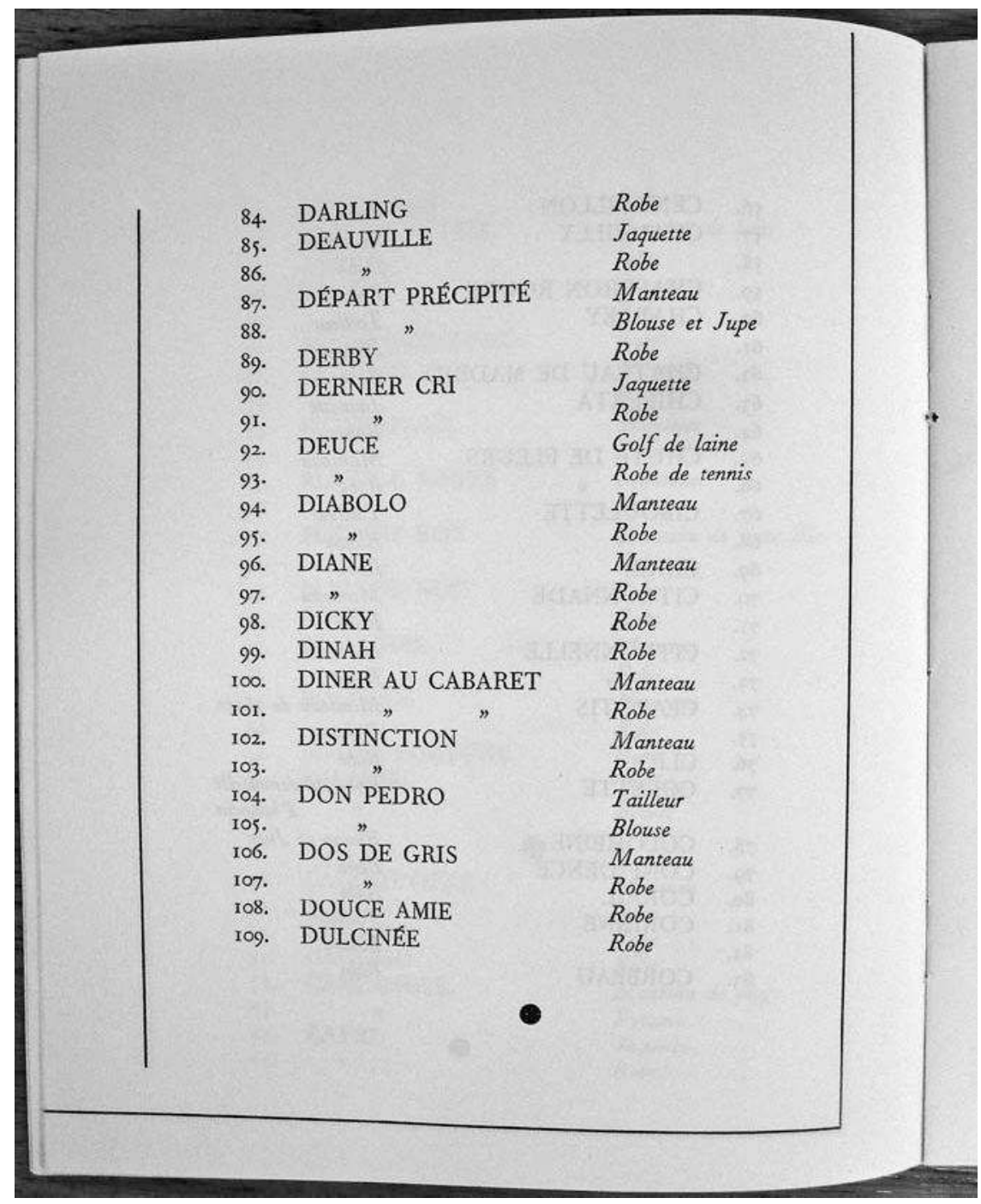

Ill. 7 : Jean Patou, Livret de défilé, été 1929, Bibliothèque Forney, CC2404. Cl. Johanna Zanon.

Car c'est bien de ça qu'il s'agit, le défilé est une accumulation de plus de trois cents robes, portées par la cabine des mannequins. Lors du défilé de février 1925 avec ses six mannequins recrutés aux États-Unis, Jean Patou rend visible la connexion avec l'esthétique industrielle implicite depuis la Belle époque ${ }^{48}$. On ne sait plus si

48. Caroline Evans, "Le défilé de mode au début du XXe siècle à Paris : esthétique industrielle et aliénation moderniste", Showtime, Paris, p. 83. 
le mannequin est un être humain ou un automate. Le couturier pousse l'uniformisation à son paroxysme en faisant défiler ses mannequins comme autant de corps standardisés à la démarche calibrée, les uns à la suite des autres vêtus du même fourreau. À l'instar des meneuses de revue étudiées par Siegfried Kracauer en 1927, les mannequins, uniques individuellement, deviennent sur le podium un "ornement de masse ${ }^{49}$ ". La ligne créée par ces unités identiques renvoie à la chaîne d'assemblage, dont Charlie Chaplin fera une brillante satire dans Les Temps modernes (1936). L'image est accentuée par les gestes mécaniques des mannequins, en écho aux méthodes tayloristes et fordistes dont s'inspire Jean Patou. Caroline Evans offre une brillante démonstration de la manière dont le défilé de février 1925 cristallise les tensions entre l'unique et le multiple, l'art et l'industrie, la France et l'Amérique, la consommation de l'élite et la production de masse ${ }^{50}$ (ill. 8).

Le carton d'invitation, le livret de défilé et le livret d'accompagnement constituent la littérature commerciale qui entoure le défilé, et en constitue sa propédeutique, en ce qu'ils préparent et conditionnent l'œil de la cliente. La succession des titres des modèles de couture offerts en pâture aux clients esbaudis, leur ordre d'apparition et leur nature s'affichent sur le livret de défilé, "sorte de programme pour une féerie en trois cents robes ${ }^{51}$ ». Les clients y repèrent la robe désirée et entourent son numéro pour l'essayer ensuite. Chez Jean Patou, cette littérature publicitaire possède son langage propre et constitue un exercice de style renouvelé selon le rituel saisonnier. La couverture de chaque livret est illustrée par une vignette gravée, dans l'esprit des gravures du XVIII e siècle. Au milieu d'un paysage nuageux, deux angelots et un putto encadrent une tablette sur laquelle est inscrite la devise de la maison : "La Mode est Reine ". À cette vignette se mêlent une composition, une typographie et un jeu des couleurs modernes. La bichromie, dans des tons propres à l'Art Déco, est la marque des livrets de Jean Patou. À l'été 1929, elle s'exprime dans des accords argenté et capucine, couleur dominante de la nouvelle collection comme en témoignent Les inséparables (capucine) ou Patou's light capucine. Par la lecture du livret, le client s'imprègne de cette couleur avant même de voir les modèles défiler. La juxtaposition sur la couverture de recherches propres à l'Art Déco et d'une référence à la gravure du XVIII ${ }^{\mathrm{e}}$ siècle interpelle. Et c'est peut-être bien là le but de cette curieuse alliance entre le moderne et l'ancien, qui se retrouve jusque dans l'architecture de la maison de couture, installée dans un immeuble du XVIII ${ }^{\mathrm{e}}$ siècle rue Saint-Florentin, mais dont l'aménagement et la décoration sont réalisés par Louis Süe et André Mare dans un style Art Déco.

49. Siegfried Kracauer, The Mass Ornament, [1927], 1995, Cambridge, Harvard University Press, 403 p., p. 78.

50. Caroline Evans, The Mechanical Smile : modernism and the first fashion shows in France and America, 1900-1929, 2013, New Haven, London, Yale University Press, p. 115-137.

51. Louis Roubaud, op. cit., p. 162-163.

Livraisons d'bistoire de l'architecture $n^{\circ} 27$ 


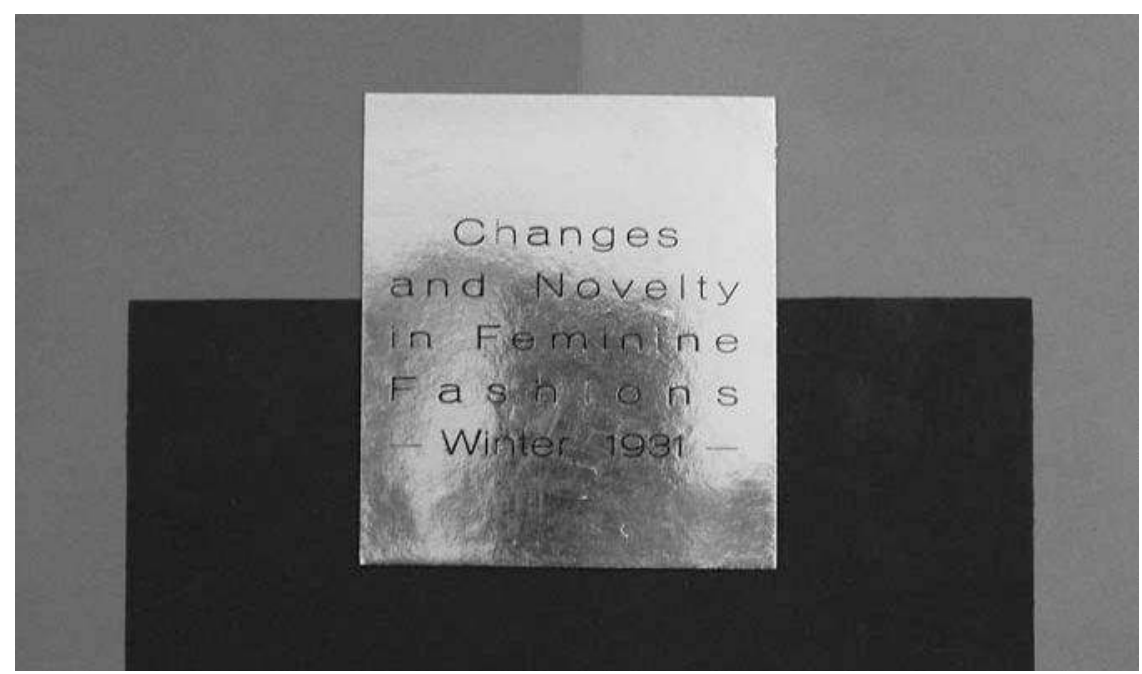

Ill. 8 : Jean Patou, Livret d'accompagnement du défilé, hiver 1931, Bibliothèque Forney, CC2404. Cl. Johanna Zanon.

\section{Les manifestes de l'élégance: le règne de la narration}

La litanie des titres de mode trouve un écho dans le livret d'accompagnement du défilé, qui met en scène la collection présentée. Jean Patou n'est cependant pas le premier couturier à accompagner ses présentations d'éléments rédactionnels. Ainsi, au début du $\mathrm{XX}^{\mathrm{e}}$ siècle, Lucile organise le défilé de ses modèles en cortèges thématiques; les plus élaborés sont mis texte par sa sœur, la romancière Elinor Glyn. Les séries de Lucile culminent en 1909 avec l'ambitieux Sept âges de la fermme, une pièce en sept actes, retraçant le cycle vestimentaire d'une élégante de la naissance à la mort, selon Nancy J. Troy ${ }^{52}$. Les livrets d'accompagnement du défilé chez Patou sont le règne de la narration, ils sont le support privilégié du storytelling de la marque élaboré par la communication. Didactiques, ils visent à éclaircir les nouvelles tendances et s'organisent tous de la même manière. Une définition générale de la collection sert d'introduction à une description plus approfondie de chaque type de vêtements et des spécificités de la saison. À l'hiver 1931, l'une d'entre elles est consacrée à l'influence perse, que l'on retrouve dans les titres de mode : le livret légitime le titre, il lui sert, en quelque sorte, de définition. Le caractère pédagogique des livrets d'accompagnement est encore sensible dans le discours employé : "Je le répète, la grande caractéristique de cette collection [...] est le retour aux couleurs et surtout à leurs mélanges ${ }^{53}$ ".

52. Nancy J. Troy, Couture Culture : A Study in Modern Art and Fashion, 2003, Cambridge, MIT Press, 438 p., p. 93.

53. Jean Patou, Livret d'accompagnement du défilé, été 1931, Bibliothèque Forney, cote CC2404. 
Au-delà, peut-être faut-il voir dans le livret d'accompagnement un "shifter ${ }^{54}$ " selon la terminologie établie par Roman Jakobson et étendue par Roland Barthes, qui permettrait la translation de la structure technologique à la structure verbale ou, pour le dire autrement, du vêtement réel au vêtement écrit. Le livret rejoint le catalogue qui « veut moins rendre compte du vêtement que persuader qu'il est à la Mode $^{55}$ ". Le livret délivre la mode selon Jean Patou ; son titre affirme le « concept de mode ${ }^{56}$ " de la maison, pour reprendre la formule de Sylvie Roy. Tous s'intitulent $D u$ Changement et des Nouveautés dans la Mode Féminine, affichant le refus des traditions. Une maxime, qui précise la philosophie de Jean Patou, accompagne le titre du livret: "Good taste is a useful quality, but to achieve real novelty you must dare" à l'été 1929 et "To-day's fashion is a living fashion" à l'été 1930. Le livret d'accompagnement tient un discours qui vise à la fois à convaincre la cliente du goût du couturier et de sa prescience de la mode à venir. À chaque saison, le livret d'accompagnement affirme avec force l'identité de la collection et de la maison, agissant comme autant de manifestes artistiques ou littéraires. Du fait de ses accointances avec ses homologues, on le nommera manifeste de la couture ou de l'élégance, malgré son but commercial évident.

Une fois le manifeste de l'élégance posé comme genre, il faut en définir sa " grammaire ${ }^{57}$ ", selon le vocable employé par la spécialiste de ce genre littéraire, Jeanne Demers. Le couturier s'implique personnellement - officiellement, à tout le moins - dans ce manifeste qui est écrit à la première personne et signé de son nom. Le discours est centré sur un double destinataire, le groupe à rallier, les élégantes, et le groupe à exclure, composé des individus qui perpétuent le mauvais goût. Sa cible à l'été 1931, par exemple, est constituée des robes de style. Pour se faire entendre, Jean Patou n'hésite pas à employer un ton autoritaire: «Mes directives pour les sports d'hiver sont : variété plus grande, éloignement du côté classique des dernières saisons, tout en restant soucieux du côté pratique ${ }^{58}$ ». De la verve agressive du manifeste artistique, toutefois, il ne reste que des échos. La légèreté et la frivolité associées à la mode à l'époque se retrouvent dans le vocabulaire imagé et le registre anecdotique :

Dans mon enfance, âge héroïque de la bicyclette, j’ai connu un homme qui ayant appris à monter et pouvant garder son équilibre, n'était jamais capable de s'arrêter. Il roulait en criant éperdument aux gens qu'il apercevait: "Arrêtez-moi! Arrêtez-moi!". L'aventure de ce brave homme est un peu la mienne $\mathrm{e}^{59}$ !

54. Roman Jakobson, Essais de linguistique générale, 1963, Paris, Éditions de Minuit, chap. 9 ; Roland Barthes, Système de la mode, Paris, Le Seuil, 1967, 330 p., p. 16.

55. Roland Barthes, ibid., p. 17.

56. Sylvie Roy, "L'invitation au défilé ", Showtime: le défilé de mode, catalogue d'exposition, Paris, Paris-Musées, 2006, p. 242.

57. Jeanne Demers, "Entre l'art poétique et le poème : le manifeste poétique ou la mort du père ", Études françaises, vol. 16, $\mathrm{n}^{\circ} 3-4,1980$, p. 10.

58. Jean Patou, Livret d'accompagnement du défilé, hiver 1930, Bibliothèque Forney, cote CC2404.

59. Loc. cit. 
Le dernier élément de cette grammaire est le sentiment d'impuissance du couturier : "C'est l'été et c'est, tout au moins chez moi, la constante offensive contre le bibi des dernières années ${ }^{60} \%$. Le manifeste de couture, à l'instar de ses homologues artistiques et littéraires, ne poursuit pas l'idée d'une mode idéale. Il contient en son sein sa propre mort à venir du fait du renouvellement permanent de la mode. En effet, la mode occidentale est constamment tiraillée entre l'imitation, concept manié par Gabriel de Tarde, et la distinction, concept de Georg Simmel ${ }^{61}$, repris ensuite par Pierre Bourdieu. Simmel déclare qu' "à chaque fois, une fraction seulement du groupe la pratique, l'ensemble ne faisant que chercher à la rejoindre ${ }^{62}$ ». L'avènement d'une mode idéale signifierait la négation même du phénomène de mode. Le manifeste de la couture se condamne donc à l'autodestruction, mais c'est peut-être aussi ce qui le rattache au genre car tout manifeste est périmé dès qu'il est énoncé ou, du moins, dès qu'il existe un horizon d'attente de ce manifeste. Le livret d'accompagnement est amené à se contester et à se contredire d'une saison sur l'autre. Il perd de sa crédibilité en tant que manifeste par sa répétition semestrielle programmée. Ces livrets ne seraient pas vraiment en opposition au système, ils constitueraient davantage "un risque calculé du système ${ }^{63}$ ", d'après Jeanne Demers. La modération, la programmation cyclique des livrets et l'absence de quête d'une mode idéale élèvent ces livrets au rang d'anti-manifestes.

Leur particularité chez Jean Patou, en outre, est de participer à une dynamique du scandale. Lorsqu'il rallonge les jupes lors de la collection hiver 1929-1930, le couturier proclame dans ses livrets d'accompagnement l'innovation dont il est l'instigateur, contribuant à forger sa propre légende. Il écrit ainsi dans l'un d'entre eux que le rallongement des jupes "marked one of the most complete revolutions that have been seen in the history of fashion ${ }^{64}$ ". Par ailleurs, il n'est pas anodin que la guerre déclarée à l'ourlet cours soit strictement contemporaine de la réédition du Manifeste du Surréalisme d'André Breton ${ }^{65}$ - bien que cela ne signifie pas que le couturier ait noué des liens avec ce mouvement réservé à un cercle d'initiés très restreint. La même force anime, en effet, ces deux entreprises, et l'on pourrait presque ajouter à la célèbre énumération d'André Breton : "Patou est surréaliste dans la promotion ». Pourtant, les pratiques du couturier seront perçues comme "very Yankee ${ }^{66}$ ». En effet, Nancy J. Troy affirme qu'une stratégie marketing opérant à autant de niveaux simultanément était presque automatiquement associée aux pratiques entrepreneuriales américaines. Il convient toutefois de nuancer cette

60. Jean Patou, Livret d'accompagnement du défilé, été 1931, Bibliothèque Forney, cote CC2404.

61. Gabriel de Tarde, Les Lois de l'imitation, étude sociologique [1890], 1993, Paris, Kimé, 428 p.; Georg Simmel, «La Mode» [1895], La Tragédie de la culture, 1993, Paris, Rivages, 254 p.

62. Georg Simmel, ibid., p. 100.

63. Jeanne Demers, op. cit., p. 6.

64. Jean Patou, Livret d'accompagnement du défilé, été 1930, Bibliothèque Forney, cote CC2404.

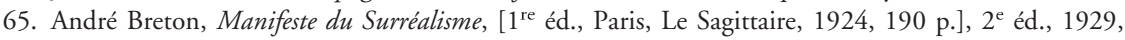
Paris, Kra, 206 p. ; André Breton, Second manifeste du Surréalisme, 1930, Paris, Kra, 103 p.

66. Vanina, "La Comédie de la mode: décentralisons... ", Comoedia Illustré, no 1, 5 octobre 1912, p. 29, cité par Nancy J. Troy, op. cit., p. 96. 
influence, somme toute assez schématique, et de ne pas minorer l'impact des autres facteurs.

Au titre de mode au manifeste de l'élégance, l'analyse du modèle onomastique de la maison Jean Patou dans l'entre-deux-guerre a montré l'importance de l'écrit qui enrobe les modèles de couture, en une sorte de cadre invisible. Les titres de mode se situent à la croisée des genres entre le titre artistique et l'accroche commerciale. Au-delà de l'évidente séduction dont ils font preuve, s'y concentrent à la fois l'appropriation des tendances de la saison et l'identité de la marque. À la lisière du vêtement, le titre est un passeur, un médiateur, un intermédiaire entre la maison de couture et la cliente. Il peut endosser ce rôle en raison de son ancrage socioculturel, témoigne du mode de vie et de consommation des élites. Ce titre de mode se modifie lorsqu'il devient titre de parfumerie et de cosmétique, justement pour toucher un public plus large, c'est grâce à cette diversification que survit le nom des grandes maisons comme Jean Patou. La diffusion des parfums et cosmétiques pose aussi la question de la langue et de la traduction du titre, en fonction des stéréotypes culturels de la France, des États-Unis et de l'Italie. Le titre n'est jamais isolé, le titre vit en communauté. Les titres inscrits les uns à la suite des autres dans le livret de défilé font écho au vertige de la liste. Le titre est encore au centre du livret d'accompagnement, qui vise à l'expliquer et à révéler les codes de la mode de Jean Patou. Que ce soit dans l'un ou dans l'autre, le titre occupe un place centrale dans la littérature commerciale, véritable propédeutique au défilé moderne, tel qu'il s'invente chez Patou à partir du milieu des années 1920.

L'étude des titres de mode, certes contrainte par la difficulté de retrouver les titres ou les modèles de couture et de les ré-associer, mériterait d'être poursuivie tant d'un point de vue chronologique que comparatif. Le champ de réflexion autour de l'onomastique commerciale demeure aussi vaste qu'inexploré.

Johanna ZANON archiviste paléographe 